\title{
A Case Study: Ingestion Analysis of WSN Data in Databases using Docker
}

\author{
W. Velásquez, member IEEE* Andres Munoz-Arcentales ${ }^{\dagger}$, Joaquín Salvachúa Rodríguez ${ }^{\ddagger}$ \\ $\ddagger$ Departamento de Ingeniería de Sistemas Telemáticos, Escuela Técnica Superior de Ingenieros de Telecomunicación \\ Universidad Politécnica de Madrid, Spain, Email: ${ }_{j} j$ salvachua@dit.upm.es \\ ${ }^{* \dagger}$ Escuela Superior Politécnica del Litoral, ESPOL, FIEC P.O. Box 09-01-5863, Guayaquil, Ecuador \\ Email: (*wavelasq, †joanmuno)@espol.edu.ec
}

\begin{abstract}
In this paper, we present an analysis of the different database systems for storing the information gather by a wireless sensor network. In this work we present different test scenarios in order to evaluate the Fetch Time and the use of Mebibyte in Relational (MySQL, PostgreSQL) and No-SQL (MongoDB, Couch, Neo4J) databases. For achieving this goal, the database instances are deployed in Docker containers for providing the same deploy, host platform and, also present a performance analysis of the resources for each database. Moreover, we conduct a case of study focus in the storage of the asynchronous information collected by a WSN in a reactive evacuation system, this is information is handled by a Kafka cluster and Zookeeper topics. Finally the tests made in this work provide the information needed for making the correct elections of the database in this type of systems.
\end{abstract}

Keywords-Kafka; wireless sensor networks; database; mysql; mongodb; couchdb; neo4j; postgresql; scala; akka; docker.

\section{INTRODUCTION}

The large-scale implementation of a Wireless Sensor Network (WSN) real-time monitoring system that allows evacuations for emergency events presents a challenge in managing all information from environmental sensors [1]. Due to the increase in Internet users, social media platforms and the size of the data generated have shown some deficiencies in traditional databases [2]. Currently, the companies want to adapt big data techniques due to the amount of information they have, among them are: Hadoop [3] or Spark Streaming [4]. These are tools that use the data to estimate or predict based on the information stored.

In the world of databases, several comparative works are presented, such as: Abramova et al [5], [6], where the report focuses mainly on the execution time of different NoSQL Databases in independent systems. In the same way, works that focus on the scalability [7] or the presented by Cooper et al., [8] where shows an analysis of latency vs. throughput tradeoff in a computer environment of different NoSQL databases. In order to control a large amount of data from a WSN, we have chosen to work with relational and nonrelational databases to compare and analyze their behavior under the virtual machine mode using Docker [9] containers.

The present work is a continuation of the proposal by Muñoz, et al. [10], where WSN is proposed for emergency situations within ESPOL's administrative building (1692 $\mathrm{m}^{2}$ ); managing in a distributed environment sensors, servers, signaling and communications. The WSN obtains data from environmental sensors such as: Temperature (TMP), Carbon Monoxide (CO), Carbon Dioxide (CO2), Petroleum Liquid Gas (PLG) and Vertical Liquid Level (VLL) to provide an optimal route evacuation in an emergency. The signaling provides a sequence of lights inside the building that will guide users to the nearest exit. In order to safeguard communication in an emergency; In the design proposed by Muñoz; Three different links are used: An Ethernet interface for communication with the server, a $3 \mathrm{G}$ link in case the Ethernet fails and a Zigbee to obtain information from the WSN. The server uses the Dijkstra algorithm to provide an evacuation route, based on information from nodes that have some drawbacks, but the use of this component is out of the scope of this document. Moreover, the verification of the model proposed by Velásquez, et al. [11], he presents a distributed model for data management using a Kafka cluster [12] as the communication core. Use Zookeeper Topics to segment data flow as well as manage alarms and turn on lights. The main objective of this work is to analyze the type of database most suitable to handle large amounts of data with an optimal response.

The present paper is divided in four sections: Section II describes the state of the art technologies used. Section III presents the schemes and configuration of the simulation. Section IV shows an analysis of the different databases and their behaviors. Finally, the paper presents a conclusion of the data analyzed based on the results obtained.

\section{STATE OF ART}

In this section, we present a study of the technologies implemented to recreate simulated scenarios, prior to the comparison and analysis of the solution, as shown in Figure 1:

\section{A. Databases}

In the world of databases, there is a great variety applied to different applications, we can classify them in: Relational Database (RDB) and Non-Relational Databases (NoSQL) [13].

- RDB: It is a set of tables that contain specific data. The table contains one or more categories of data in columns. Each row in a table contains a data instance of 


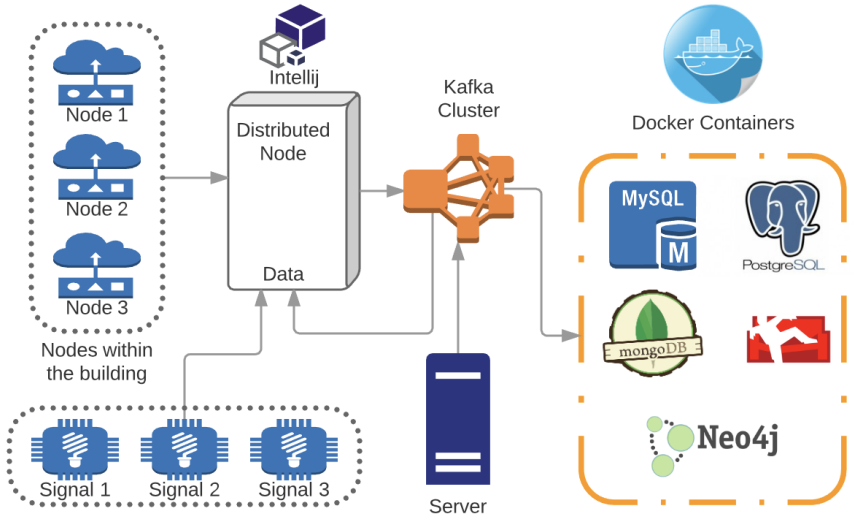

Figure 1. Architecture of a wireless sensor network with a centralized cluster

an object. Relationships can be established between the tables to resemble the real world [14]. In this analysis, we focus on the MySQL [15] and PostgreSQL [16] databases.

- NoSQL: These types of databases present a structured storage, depending on the way in which they store the data, they can be in: documents, columns, key/value, multi-value and oriented to graphs [17]. In this analysis, we focus on the MongoDB [18], Apache CouchDB (Based on Documents) [19] and Neo4j (Graph Database) [20].

\section{B. Docker Machine}

This technology allows to create a system that houses several Docker containers, allowing them to work under the same network and to interact with each other, even between applications. In this environment, it is used to create different containers from the databases described in the previous subsection. In addition, the main reasons for using this technology is due to the advantages it has compared to traditional virtual machines [21]:

- Application deployment: Containers include the minimal runtime requirements, reducing their size and allowing them to be deployed quickly.

- Portability: An application and all its dependencies can be bundled into a single container that is independent from the host version of Linux kernel, platform distribution, or deployment model.

- Lightweight footprint: Docker images are very small, which facilitates rapid delivery and reduces the time to deploy application.

\section{Communication}

The core of the communication is the Kafka Cluster, which uses Zookeeper topics for each metric, in order to provide segmentation to the data flow and easy access of the applications.
To establish communication between all elements of the network, the following links have been configured:

- Using Akka [22] in Scala [23], we have: (Sensors Distributed Node), (Reactive Signaling - Distributed Node).

- In the links (Distributed Node - Kafka Cluster), (Kafka Cluster - Docker Containers). Producers and consumers programmed in Scala are used to transmit and store information.

\section{Simulation ENVIRONMENT}

In this section, the configurations and diagrams necessary for the execution of the environment are presented. Table I shows the software used and a short description of its use.

Table I

SOFTWARE USED IN THE SIMULATION ENVIRONMENT

\begin{tabular}{|c|c|c|}
\hline Software & Version & Used for \\
\hline MacOS Sierra & 10.12 & $\begin{array}{l}\text { An environment where the simulation } \\
\text { is run }\end{array}$ \\
\hline Intellij IDEA CE & 2016.3 & Programming the different modules \\
\hline Docker machine & 0.8 .2 & $\begin{array}{l}\text { Run the docker containers from the } \\
\text { different databases }\end{array}$ \\
\hline VirtualBox & 5.1 .10 & Used by Docker machine \\
\hline PgAdmin & 4.0 & PostgreSQL Database Management \\
\hline MySQL Workbench & 6.3 & MySQL Database Management \\
\hline MongoDB Compass & 1.5 .1 & MongoDB Database Management \\
\hline Kafka Cluster & 2.0 & Content distributor \\
\hline
\end{tabular}

\section{A. Sensor Nodes}

Being $n \rightarrow$ Sensor nodes, $t \rightarrow$ Time, $s g \rightarrow$ Signaling, $t s \rightarrow$ Temperature sensor, $c s \rightarrow$ Carbon monoxide sensor, $x s \rightarrow$ Carbon dioxide sensor, $p s \rightarrow$ Petroleum liquid gas sensor and $v s \rightarrow$ Vertical liquid level sensor, and $n=30$ (This has been considered to cover each point at the building). It is true that $\forall n: P(f(t)) \rightarrow f(t)$ obtains values about $t s \in Z: 22 \leq t s \leq 25 ; c s \in Z: 20 \leq c s \leq 26 ; x s \in$ $Z: 300 \leq x s \leq 330 ; p s \in Z: 135 \leq p s \leq 295$ and $v s \in Z: 0 \leq v s \leq 5$ each $t=5 s$ (the equation is presented later). Moreover, $\forall n$ sends data to the port 25555 following the format: id_node : type_sensor $(t s, c s, x c, p s, v s)$ : value : checksum, where each of the data means the following:

- ID Node: Identifier of the node that sends this information

- Type Sensor: Type of sensor sending the data

- Sensed value: This is the value sensed by each of the sensors in the network, this value will depend on the sensor.

- Checksum: Responsible for validating the frame.

\section{B. Kafka Cluster}

Kafka is listening on port 9092 and the Zookeeper on port 2181, both ports by default. Moreover, the topics described in Table II are set up to manage producers and consumers in the simulation. 
Table II

TOPICS CONFIGURED IN THE KAFKA CLUSTER

\begin{tabular}{lll}
\hline Topic & Meaning & Package received from \\
\hline TMP & Temperatura & Distributed Node \\
CO & Carbon Monoxide & Distributed Node \\
CO2 & Carbon Dioxide & Distributed Node \\
PLG & Petroleum Liquid Cas & Distributed Node \\
VLL & Vertical Liquid Level & Distributed Node \\
AL & Alarm & Distributed Node \\
SG & Signal & Server \\
DB & Database & Distributed Node \\
\hline
\end{tabular}

\section{Data Flow}

Based on the Kafka Cluster configuration. A node has been designed to manage producers and consumers of each metric, as shown in Figure 2:

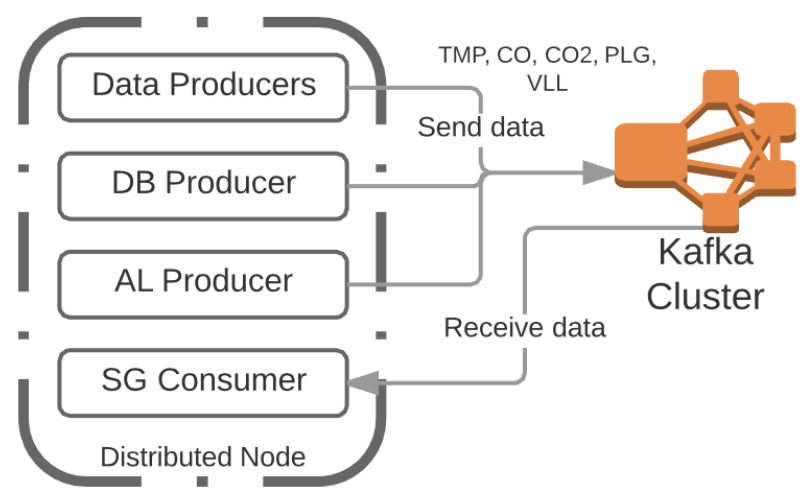

Figure 2. Producers and Consumers responsible for the flow of data to Kafka

- Data Producers (TMP, CO, CO2, PLG, VLL): Responsible for data information from the nodes and transmitting it to the producers of the Kafka cluster.

- DB Producer: This producer is in charge to transmit the data producers information to the databases.

- AL Producer: When data are obtained from the sensors, the node processes them to determine if there are any drawbacks with the thresholds that have been configured for each metric. If there were any anomalies, an "Alarm" packet is sent in the format: id_node : status : checksum

- ID Node: Node identifier with some problem

- Status: A status value is sent to identify the alarm priority (0: low, 1: medium, 2: high)

- Checksum: Responsible for validating the frame.

- SG Consumer: This consumer will be in charge of processing the information sent by the server for the signaling activation.

For the distributed node design, the architecture (Master $\rightarrow$ Slave) [22] has been considered, as shown in Figure 3:

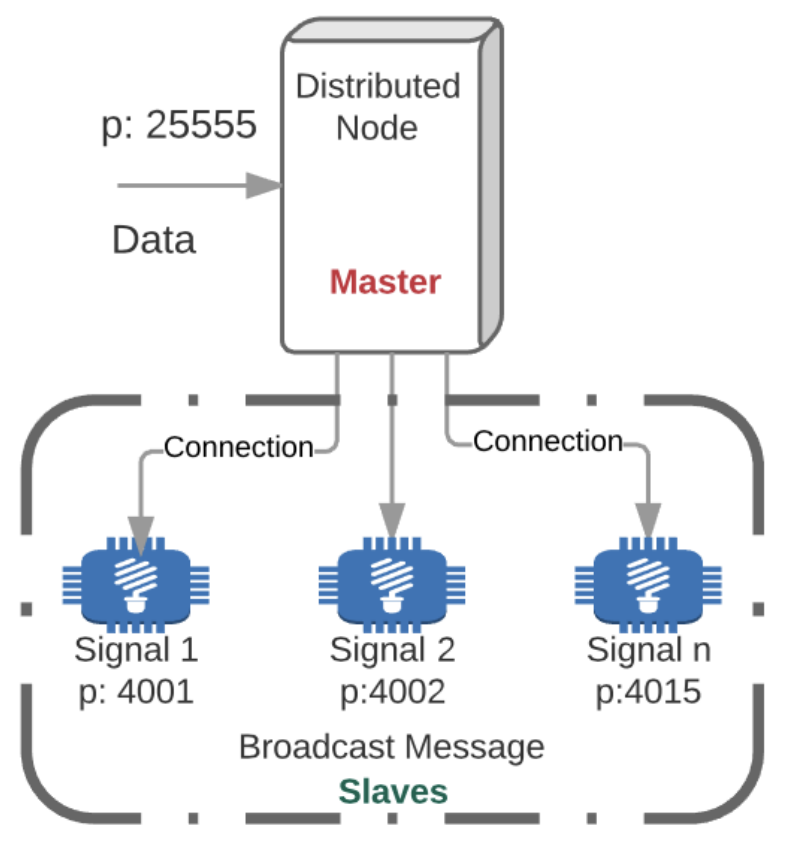

Figure 3. Architecture (Master $\rightarrow$ Slave) used by the Distributed Node programmed using Akka

According with the previously description of the system, we can determine that, $\forall n$ sends data to $p: 25555$, the distributed node is listening to this port, then, it classifies them according to the data type. When a packet is processed, it is transmitted to the Kafka cluster by checking the specific topic of each metric. Moreover, thresholds have been set for each data type, as follows: $t s \leq 40\left({ }^{\circ} \mathrm{C}\right)$, cs $\leq 200(\mathrm{ppm})$, $x s \leq 400(\mathrm{ppm}), \mathrm{ps} \leq 750(\mathrm{ppm})$ and $v s \leq 10(\mathrm{~cm})$. If a value passes the thresholds, a packet is sent to the AL topic, so that the server can process it and check for an emergency. Therefore, if there is a problem, the server sends a packet to the SG topic, so that the distributed node obtains it and re-transmits it to the slaves (signaling) to turn on the lights.

\section{Signaling}

If $s g=15$, it is true that $\forall s g$ has an actor listening in a port $p: p \in Z: 4001 \leq p \leq 4015$. When an emergency occurs, the distributed node sends a packet in the format: id_signal : status : address : checksum.

$\forall s g$ receives the package and processes it to verify the status of emergency \{status $\rightarrow$ [warning : 00,danger : $01]\}$ and the direction to turn on the lights $\{$ address $\rightarrow$ [right: 00, left: 01]\}.

\section{E. Docker Containers}

The docker containers help us to facilitate the installation work of the databases that are going to be evaluated. Because we can deploy an image with all the configuration needed to build the test environment and configure the containers with 
the same network connection in order to communicate the containers between them. Table 3 shows the configurations of the databases:

Table III

DOCKER CONTAINERS WITH THE VERSION OF THE DATABASE

\begin{tabular}{lcl}
\hline \multicolumn{1}{c}{ Database } & $\{$ Version $\}$ & \multicolumn{1}{c}{ IP address:Port } \\
\hline MySQL & 5.7 .17 & $192.168 .99 .100: 3306$ \\
PostgreSQL & 9.6 .1 & $192.168 .99 .100: 5432$ \\
MongoDB & 3.4 .1 & $192.168 .99 .100: 27017$ \\
Apache CouchDB & 1.6 .1 & $192.168 .99 .100: 5984$ \\
Neo4j & 3.1 .0 & $192.168 .99 .100: 7687$ \\
\hline
\end{tabular}

The dockers are configured under the command line as follows: docker run - $p$ \{IP Address:Port $\}$-detach name $=\{$ Container Name $\}-e-d\{$ Database:Version $\}$

\section{F. Databases Model}

In this section, the models implemented in the databases are described:

1) Relational Database Model: Table IV shows the relational model implemented in the MySQL and PostgreSQL database. In this design, a specific table for each metric (TMP, CO, CO2, PLG, VLL) has been declared due to the amount of data stored by the WSN. Moreover, a table to store the alerts caused in the simulation and one of the signals that are activated.

Table IV

Relational MODEL of DATABASES: MySQL AND PostGRESQL

\begin{tabular}{lll}
\hline \multicolumn{1}{c}{ Table } & \multicolumn{1}{c}{ Columns } & \multicolumn{1}{c}{ Description } \\
\hline $\begin{array}{l}\text { node } \\
\text { node_co } \\
\text { node_co } 2 \\
\text { node_tmp } \\
\text { node_plg } \\
\text { node_vll }\end{array}$ & $\begin{array}{l}\text { id,status and } \\
\text { lastupdate }\end{array}$ & $\begin{array}{l}\text { Contains the nodes in } \\
\text { and ne network. }\end{array}$ \\
signal & $\begin{array}{l}\text { id signal, status, } \\
\text { address and date } \\
\text { id, id node, type } \\
\text { and date }\end{array}$ & $\begin{array}{l}\text { Stores the values of } \\
\text { each metric. } \\
\text { occur in an emergency } \\
\text { Stores the alarms that are } \\
\text { produced by the nodes. }\end{array}$ \\
\hline
\end{tabular}

2) NoSQL Database - Based on Documents: The NoSQL database model based on documents from the MongoDB and Apache CouchDB databases is presented in Table V. Due to the way in which these databases are handled, for each record a new document will be created, having redundant data coming from the nodes.

3) NoSQL Database - Neo4j: This database schema is based on the model of a graph, for that reason; Nodes and relationships have been declared, as shown in Figure 4. Each of the nodes (TMP, CO, CO2, PLG, VLL) will have a value field in the node and a date field in relation to the node. Similarly, the alarm node will have the date field, with the difference that it will be one of the relation.
Table V

Document Model For the MongoDB And Apache Couch DB DATABASES

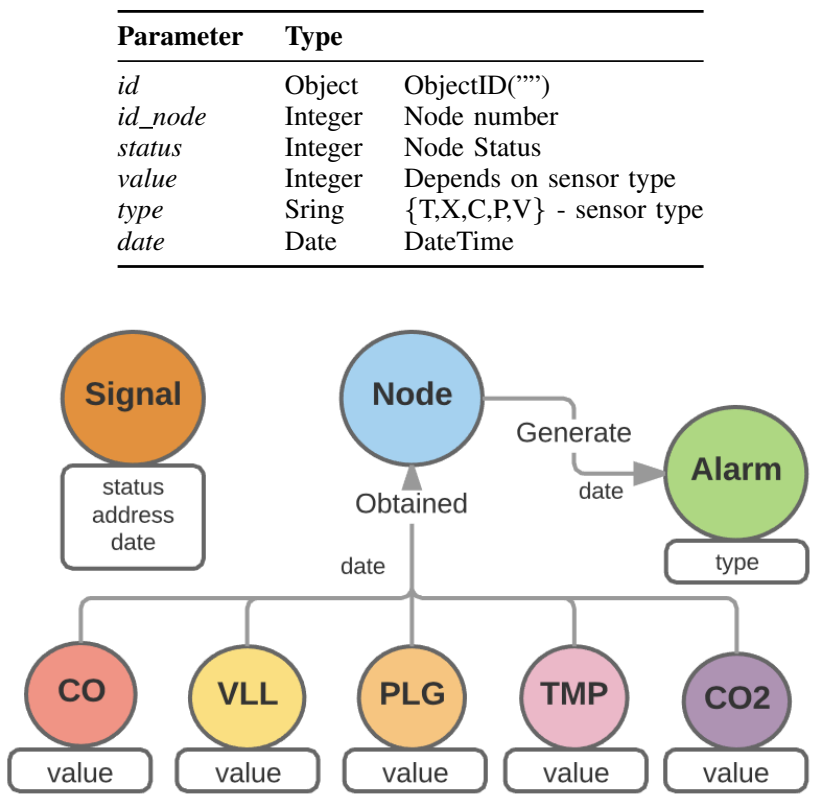

Figure 4. Graph-oriented database model - Neo4j

\section{G. Experimental Setup}

The scope of the experiments can be viewed in Table VI. A total of 3 (Duration Time) $* 5$ (Databases) $* 5$ (Node on Alert $)=75$ experiments were conducted to know the throughput of the database on the simulation.

Table VI

TEST SCEnARIOS

\begin{tabular}{ccc}
\hline Time (h) & Databases & Nodes on alert (\%) \\
\hline 1 & MySQL & 0 \\
3 & PostgreSQL & 10 \\
5 & MongoDB & 30 \\
& CouchDB & 50 \\
& Neo4j & 70 \\
\hline
\end{tabular}

In addition, It must be considered that the activation of the nodes $n$ is sequential so there is a delay $\tau$ between them at the time of data capture. The sampling can be expressed by the following expression:

$$
\text { data }=n_{1} x(t)+\sum_{i=2}^{30} n_{i} x\left(t+\sum_{j=1}^{i} \tau^{\prime} j\right)
$$

Given that $n_{1}$ is the first to be power does not have a delay $\tau$, on the other hand, other nodes are accumulated power of the previous delays $x(t+\tau)$.

\section{AnAlysis OF RESUlts}

This section evaluates the results of each of the databases for the different scenarios. We present our analysis on the 
throughput and the reasoning contributing to the behavior of each system.

\section{A. Database Performance}

In Figures 5, 6 and 7, we show the average of the results of all the node alert cases for the different duration scenarios. Where, it is appreciated that the Neo4j database has the best time (Fetch Time) when making a query and MySQL the least use of Mebibytes (MB) in storage followed by MongoDB.

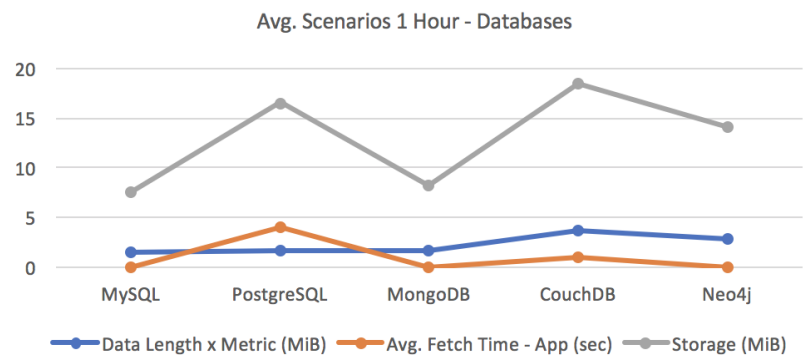

Figure 5. Database performance on the stage 1 hour

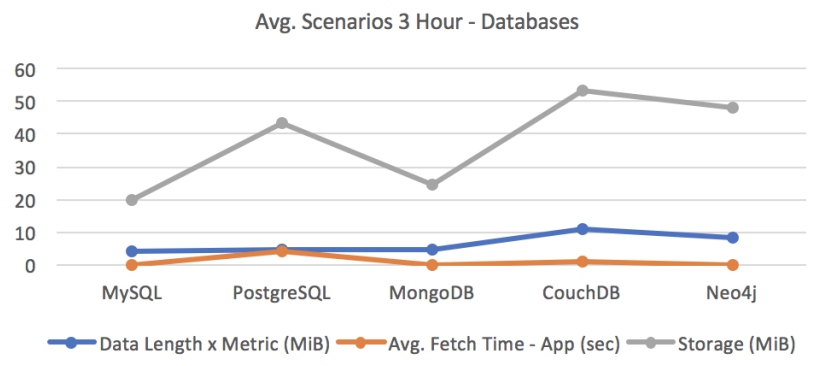

Figure 6. Database performance on the stage 3 hour

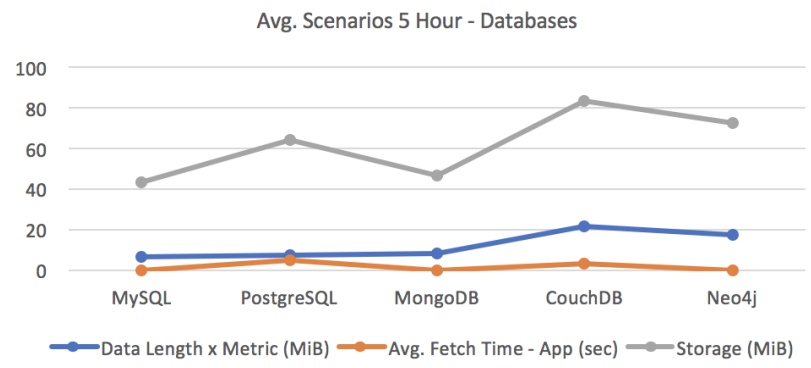

Figure 7. Database performance on the stage 5 hour

Moreover, Table VII shows the average of the amount of data that is inserted in the databases in the hour-long scenario. The PostgreSQL database presents the largest amount of data stored as a function of the transmitted data. On the other hand, Couchdb presents the worst performance in data ingest and storage size, because of the $100 \%$ of packets transmitted in the maximum duration time could only store $28,2 \%$ of the data.

Table VII

DATA INSERTED AND TRANSMITTED IN DATABASES - CASE ONE HOUR

\begin{tabular}{lccc}
\hline Databases & Transmitted & Stored & Received DB Topic \\
\hline MySQL & 20935 & 16192 & 104677 \\
PostgreSQL & 21091 & 16347 & 105455 \\
MongoDB & 20980 & 16237 & 104900 \\
CouchDB & 20995 & 5381 & 104979 \\
Neo4j & 21264 & 15739 & 106324 \\
\hline
\end{tabular}

Analyzing deeply the results of Table VII, it is noticed that due to the alarms in the nodes and given the priority when sending the notifications of activation of lights, a delay occurs when the data are inserted in the different databases. But, it is important to say that $100 \%$ of packets alarms are received by the slaves and the AL topic for activation signaling.

\section{B. Containers}

In Figure 8 and Table VIII, information is presented on the use of the containers by the different database systems. As a result, wsn-mongo has a low CPU processing, wsnpostgres has the lowest memory usage by $85.63 \%$ and its system size being $0.236 \mathrm{~KB}$. On the other hand, wsn-neo4j is the one with the highest value in memory usage and size in the system.

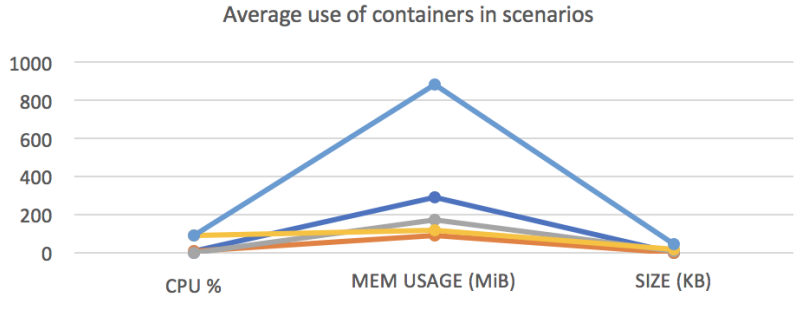

Figure 8. Average of the use of the containers in the scenarios during the simulation

Table VIII

USE OF CONTAINER SERVICES

\begin{tabular}{lccc}
\hline \multicolumn{1}{c}{ Containers } & MEM \% & NET I/O & BLOCK I/O \\
\hline wsn-mysql & 14.24 & $12.87 / 18.43 \mathrm{MB}$ & $413.7 \mathrm{kB} / 611.5 \mathrm{MB}$ \\
wsn-postgres & 4.28 & $22.46 / 8.46 \mathrm{MB}$ & $22.26 / 597.5 \mathrm{MB}$ \\
wsn-mongo & 9.11 & $25.8 / 12.39 \mathrm{MB}$ & $59.63 / 165.7 \mathrm{MB}$ \\
wsn-couch & 5.7 & $297 \mathrm{MB} / 2.9 \mathrm{~GB}$ & $14.8 / 23.6 \mathrm{MB}$ \\
wsn-neo4j & 44.05 & $11.8 / 3.2 \mathrm{MB}$ & $74.35 \mathrm{MB} / 225.3 \mathrm{kB}$ \\
\hline
\end{tabular}

\section{Discussions}

Based on the results obtained from the simulation, it can be indicated that the best database of the analyzed ones for 
the ingestion of data per second is PostgreSQL, but, for the amount of storage that occupies in $\mathrm{MiB}$ the displaced to the second place making MongoDB get better performance. This is because the important thing about the design of this WSN is that $100 \%$ of the packages arrive at the Kafka cluster and in each scenario, this premise was fulfilled, even in the transmission of the alarm packages. On the other hand, the Neo4j database stands out when making queries, but; Most WSN data are accessible in history mode and do not represent a strong point and worse considering the amount of storage that is generated, it has been discarded this database, resulting the another databases such as CouchDB do not help this kind of scenarios.

In addition, analyzing that the amount of data inserted in the databases for each time of duration is considerable, it has been proposed to separate the data ingestion with those transmitted to the Kafka cluster, resulting in each $t=5 \mathrm{~s}$ the WSN transmits to the cluster and each $\mathrm{t}=5 \mathrm{~m}$ is stored in the database causing a reduction of $70 \%$ in storage usage. On the other hand, the use of Docker containers facilitated the deployment of the different databases because the container image was only downloaded and executed, causing a low memory consumption and less server processing.

\section{ACKNOWLEDGMENT}

Thanks to the Escuela Superior Politécnica del Litoral (ESPOL) and to the Secretaría Nacional de Educación Superior, Ciencia, Tecnología e Innovación (SENESCYT) for financing the continuity of reactive evacuation systems study in emergencies.

\section{REFERENCES}

[1] J. A. Munoz, V. Calero, I. Marin, P. Chavez, and R. Perez, "Adaptive evacuation management system based on monitoring techniques," Latin America Transactions, IEEE (Revista IEEE America Latina), vol. 13, no. 11, pp. 3621-3626, 2015.

[2] P. P. Srivastava, S. Goyal, and A. Kumar, "Analysis of various nosql database," in Green Computing and Internet of Things (ICGCIoT), 2015 International Conference on. IEEE, 2015, pp. 539-544.

[3] C. Lam, Hadoop in action. Manning Publications Co., 2010.

[4] Z. Han and Y. Zhang, "Spark: A big data processing platform based on memory computing," in Parallel Architectures, Algorithms and Programming (PAAP), 2015 Seventh International Symposium on. IEEE, 2015, pp. 172-176.

[5] V. Abramova and J. Bernardino, "Nosql databases: Mongodb vs cassandra," in Proceedings of the international $C^{*}$ conference on computer science and software engineering. ACM, 2013, pp. 14-22.

[6] V. Abramova, J. Bernardino, and P. Furtado, "Which nosql database? a performance overview," Open Journal of Databases (OJDB), vol. 1, no. 2, pp. 17-24, 2014.

[7] — "Testing cloud benchmark scalability with cassandra," in Services (SERVICES), 2014 IEEE World Congress on. IEEE, 2014, pp. 434-441.
[8] B. F. Cooper, A. Silberstein, E. Tam, R. Ramakrishnan, and R. Sears, "Benchmarking cloud serving systems with ycsb," in Proceedings of the 1st ACM symposium on Cloud computing. ACM, 2010, pp. 143-154.

[9] J. Turnbull, The Docker Book: Containerization is the new virtualization. James Turnbull, 2014.

[10] A. Munoz-Arcentales, W. Yánez-Pazmino, and W. V. Vargas, "Proposal of a communication structure model for activating reactive signaling in an emergency evacuation systems," in 2017 IEEE 7th Annual Computing and Communication Workshop and Conference (CCWC), Jan 2017, pp. 1-5.

[11] W. V. Vargas, A. Munoz-Arcentales, and J. S. Rodríguez, "A distributed system model for managing data ingestion in a wireless sensor network," in 2017 IEEE 7th Annual Computing and Communication Workshop and Conference (CCWC), Jan 2017, pp. 1-5.

[12] N. Garg, Learning Apache Kafka. Packt Publishing Ltd, 2015.

[13] W. A. V. Vargas, "Bases de datos orientadas a grafos y su enfoque en el mundo real."

[14] C. Date, The relational database dictionary. Apress, 2008.

[15] N. Gehani, The Database Book: Principles \& Practice Using MySQL. Silicon Press, 2011.

[16] R. O. Obe and L. S. Hsu, PostgreSQL: Up and Running: A Practical Introduction to the Advanced Open Source Database. " O'Reilly Media, Inc.", 2014.

[17] E. Redmond and J. R. Wilson, Seven databases in seven weeks: a guide to modern databases and the NoSQL movement. Pragmatic Bookshelf, 2012.

[18] K. Banker, MongoDB in action. Manning Publications Co., 2011.

[19] J. C. Anderson, J. Lehnardt, and N. Slater, CouchDB: the definitive guide. " O'Reilly Media, Inc.", 2010.

[20] A. Vukotic, N. Watt, T. Abedrabbo, D. Fox, and J. Partner, Neo4j in action. Manning, 2015.

[21] C. Anderson, "Docker [software engineering]," IEEE Software, vol. 32, no. 3, pp. 102-c3, 2015.

[22] M. Gupta, Akka essentials. Packt Publishing Ltd, 2012.

[23] P. Haller and F. Sommers, Actors in Scala. Artima Incorporation, 2012. 